Article

\title{
Existence Results for a New Class of Boundary Value Problems of Nonlinear Fractional Differential Equations
}

\author{
Meysam Alvan ${ }^{1}$, Rahmat Darzi ${ }^{2}$ and Amin Mahmoodi ${ }^{3, *}$ \\ 1 Department of mathematics, Central Tehran Branch Islamic Azad university, Tehran 13185/768, Iran; \\ m.alvan.r.math.t@gmail.com \\ 2 Department of Mathematics, Neka Branch Islamic Azad University, Neka 48411-86114, Iran; \\ r.darzi@iauneka.ac.ir \\ 3 Department of mathematics, Central Tehran Branch Islamic Azad univesity, Tehran 13185/768, Iran \\ * Correspondence: a_mahmoodi@iauctb.ac.ir; Tel.: +98-21-6693-3501
}

Academic Editor: Hari M. Srivastava

Received: 27 December 2015; Accepted: 25 February 2016; Published: 4 March 2016

Abstract: In this article, we study the following fractional boundary value problem

$$
\begin{gathered}
{ }^{c} D_{0^{+}}^{\alpha} u(t)+2 r^{c} D_{0^{+}}^{\alpha-1} u(t)+r^{2}{ }^{c} D_{0^{+}}^{\alpha-2} u(t)=f(t, u(t)), \quad r>0, \quad 0<t<1, \\
u(0)=u(1), \quad u^{\prime}(0)=u^{\prime}(1), \quad u^{\prime}(\xi)+r u(\xi)=\eta, \quad \xi \in(0,1)
\end{gathered}
$$

Where $2 \leqslant \alpha<3,{ }^{c} D_{0^{+}}^{\alpha-i}(i=0,1,2)$ are the standard Caputo derivative and $\eta$ is a positive real number. Some new existence results are obtained by means of the contraction mapping principle and Schauder fixed point theorem. Some illustrative examples are also presented.

Keywords: Fractional boundary value problem; Contraction mapping principle; Schauder fixed point theorem; Mathematics Subject Classification 2000; 26A33; 34A08; 34B18

\section{Introduction}

In the recent years, fractional calculus has been one of the most interesting issues that have attracted many scientists, specially in mathematics and engineering sciences. Many natural phenomena can be presented by boundary value problems of fractional differential equations. Many authors in different fields such as chemical physics, fluid flows, electrical networks, viscoelasticity, try to model these phenomena by boundary value problems of fractional differential equations [1-4]. To achieve extra information in fractional calculus, specially boundary value problems, readers can refer to valuable papers or books [5-27].

In this paper, we investigate the existence and uniqueness of solution for the following new class of fractional boundary value problem

$$
{ }^{c} D_{0^{+}}^{\alpha} u(t)+2 r^{c} D_{0^{+}}^{\alpha-1} u(t)+r^{2}{ }^{c} D_{0^{+}}^{\alpha-2} u(t)=f(t, u(t)), \quad r>0, \quad 0<t<1
$$

with the boundary conditions

$$
u(0)=u(1), \quad u^{\prime}(0)=u^{\prime}(1), \quad u^{\prime}(\xi)+r u(\xi)=\eta, \quad \xi \in(0,1)
$$

where ${ }^{c} D_{0^{+}}^{\alpha-i}(i=0,1,2)$ are the standard Caputo derivative and $f:[0,1] \times \mathbb{R} \rightarrow \mathbb{R}$ is a continuously differentiable function satisfying the following assumptions: 
$\left(A_{0}\right) f \in C([0,1] \times \mathbb{R}, \mathbb{R})$ and there exists a constant $L>0$ so that

$$
|f(t, u)-f(t, v)| \leqslant L|u-v|, \quad t \in(0,1), \quad \forall u, v \in \mathbb{R},
$$

in which $L$ satisfies the condition $L<\frac{r^{2} G(\alpha-1)}{2 e^{r}}$.

$\left(A_{1}\right) f \in C([0,1] \times \mathbb{R}, \mathbb{R}), p \in C([0,1])$ and $A$ is a constant, so that

$$
|f(t, u)| \leqslant p(t)+A|u|, \quad t \in(0,1), \quad \forall u \in \mathbb{R},
$$

it satisfies the condition $0<A<\frac{r^{2} G(\alpha-1)}{2}$.

Because the boundary conditions $u(0)=u(1)$ and $u^{\prime}(0)=u^{\prime}(1)$ in (1.2) involve periodicity, it is not possible to directly transform the boundary value into integral equation. To overcome this problem, presenting a suitable substitution is needed. It is worth saying that Lemma 2.7 (see Lemma 2.3 in [17] and Lemma 2.6 in [21]) is an important and valuable tool to achieve the new result. The contraction mapping principle and fixed point theorem play the main role in finding new existence results for the problem.

The main result of this paper can be seen in two Theorems; 3.1 and 3.2. In Theorem 3.1, the uniqueness of solution is proved by using Banach contraction principle. In Theorem3.2, we present an existence theorem by means of Schauder fixed point theorem.

We can extend the result even for the following boundary value problem

$$
\sum_{k=0}^{n-1}\left(\begin{array}{c}
n-1 \\
k
\end{array}\right) r^{k c} D_{0^{+}}^{\alpha-k} u(t)=f(t, u(t)), \quad r>0, \quad 0<t<1
$$

where $n-1 \leqslant \alpha<n, n>4$, with the boundary conditions

$$
\left\{\begin{array}{cl}
u(0)=u(1), \quad u^{\prime}(0)=u^{\prime}(1), \ldots, \quad u^{(n-2)}(0)=u^{(n-2)}(1) \\
\sum_{0}^{n-2}\left(\begin{array}{c}
n-2 \\
k
\end{array}\right) r^{k} u^{n-k-2}(\xi)=\eta, \quad r>0, \quad \xi \in(0,1)
\end{array}\right.
$$

The plan of this paper is as follows:

In Section 2, we give some basic definitions and technical lemmas. Section 3 contains the proofs of our main results. Finally, we provide two examples to show the applicability of the results.

\section{Basic Definitions and Preliminaries}

In this section, we present some definitions and technical lemmas which will be used in the remainder of this paper. These and the related results and proofs can be found in the literature $[6-8,17,21]$.

Definition 2.1. $([7,8])$ The Riemann-Liouville fractional integral of order $\alpha>0$, of a function $u: \mathbb{R}^{+} \rightarrow \mathbb{R}$ is defined by

$$
I_{0^{+}}^{\alpha} u(t)=\frac{1}{G(\alpha)} \int_{0}^{t}(t-s)^{\alpha-1} u(s) d s, \quad n-1<\alpha \leqslant n
$$

whenever the right-hand side is defined on $\mathbb{R}^{+}$.

Definition 2.2. ([7,8]) The Riemann-Liouville fractional derivative of order $\alpha>0$, of a function $u: \mathbb{R}^{+} \rightarrow \mathbb{R}$ is given by

$$
D_{0^{+}}^{\alpha} u(t)=\frac{1}{G(n-\alpha)} \frac{d^{n}}{d t^{n}} \int_{0}^{t}(t-s)^{n-\alpha-1} u(s) d s, \quad n-1<\alpha \leqslant n
$$


where $n=[\alpha]+1$, and $[\alpha]$ denotes the integer part of real number $\alpha$.

Definition 2.3. ([7,8]) The Caputo fractional derivative of order $\alpha>0$, of a function $u: \mathbb{R}^{+} \rightarrow \mathbb{R}$ is defined by

$$
{ }^{c} D_{0^{+}}^{\alpha} u(t)=\frac{1}{G(n-\alpha)} \int_{0}^{t}(t-s)^{n-\alpha-1} u^{(n)}(s) d s, \quad n-1<\alpha \leqslant n
$$

whenever the right-hand side is defined on $\mathbb{R}^{+}$.

Definition 2.4. ([7,8]) The Caputo fractional derivative of order $\alpha>0$, of a function $u: \mathbb{R}^{+} \rightarrow \mathbb{R}$ is defined via the Riemann-Liouville fractional derivative by

$$
\left({ }^{c} D_{0^{+}}^{\alpha} u\right)(t)=\left(D_{0^{+}}^{\alpha}\left[u(s)-\sum_{k=0}^{n-1} \frac{u^{(k)}\left(0^{+}\right)}{k !} s^{k}\right]\right)(t)
$$

Where $n=\alpha$ for $\alpha \in \mathbb{N} ; n=[\alpha]+1$ for $\alpha \notin \mathbb{N}$.

Lemma 2.5. ([6]) Let $l \in \mathbb{N}, \alpha>0$. If $\left(D_{0^{+}}^{\alpha} u\right)(t)$ and $\left(D_{0^{+}}^{\alpha+l} u\right)(t)$ exist, then

$$
\left(D^{l} D_{0^{+}}^{\alpha} u\right)(t)=\left(D_{0^{+}}^{\alpha+\mathrm{l}} u\right)(t)
$$

Lemma 2.6. ([6]) Let $n \in \mathbb{N}, \alpha \in(n-1, n]$. If $u \in C^{n}[0, b)(b>0$ is real number), then

$$
\left(I_{0^{+}}^{\alpha}{ }^{c} D_{0^{+}}^{\alpha} u\right)(t)=u(t)-\sum_{k=0}^{n-1} \frac{u^{(k)}\left(0^{+}\right)}{k !} t^{k}
$$

holds on $(0, b)$.

Lemma 2.7. ([17,21]) Let $n \in \mathbb{N}, \alpha \in(n-1, n]$. If $u \in C^{n-1}[0, b)$ and ${ }^{c} D_{0^{+}}^{\alpha} u \in C(0, b)$, then

$$
\left(I_{0^{+}}^{\alpha}{ }^{c} D_{0^{+}}^{\alpha} u\right)(t)=u(t)-\sum_{k=0}^{n-1} \frac{u^{(k)}\left(0^{+}\right)}{k !} t^{k}
$$

holds on $(0, b)$.

Lemma 2.8. Let $r>0, g \in C[0,1]$. If $u \in C^{3}[0,1]$ is a solution of $B V P$

$$
\begin{gathered}
{ }^{c} D_{0^{+}}^{\alpha} u(t)+2 r^{c} D_{0^{+}}^{\alpha-1} u(t)+r^{2}{ }^{c} D_{0^{+}}^{\alpha-2} u(t)=g(t), \quad r>0, \quad 0<t<1 \\
u(0)=u(1), \quad u^{\prime}(0)=u^{\prime}(1), \quad u^{\prime}(\xi)+r u(\xi)=\eta, \quad \xi \in(0,1)
\end{gathered}
$$

Then $v$ satisfies

$$
\begin{aligned}
& v(t) \\
& =\frac{\eta}{r} e^{r t}-\frac{e^{r(t-\xi)}}{r\left(e^{r}-1\right) \Gamma(\alpha-2)} \int_{0}^{1} \int_{0}^{m} e^{r t}(m-\tau)^{\alpha-3} g(\tau) d \tau d m \\
& -\frac{e^{r(t-\xi)}}{r \Gamma(\alpha-2)} \int_{0}^{\xi} \int_{0}^{m} e^{r t}(m-\tau)^{\alpha-3} g(\tau) d \tau d m \\
& +\frac{1+t\left(e^{r}-1\right)}{\left(e^{r}-1\right)^{2} \Gamma(\alpha-2)} \int_{0}^{1} \int_{0}^{m} e^{r t}(m-\tau)^{\alpha-3} g(\tau) d \tau d m \\
& +\int_{0}^{1} \int_{0}^{1} \int_{0}^{m} \frac{e^{r t}(m-\tau)^{\alpha-3}}{\left(e^{r}-1\right) \Gamma(\alpha-2)} g(\tau) d \tau d m d n \\
& +\int_{0}^{t} \int_{0}^{n} \int_{0}^{m} \frac{e^{r t}(m-\tau)^{\alpha-3}}{\Gamma(\alpha-2)} g(\tau) d \tau d m d n
\end{aligned}
$$

Conversely, if $v(t)$ is given by (14), then $u=v e^{-r t} \in C^{2}[0,1]$ and $u$ is a solution of $B V P(12)-(13)$. 
Proof. Let $u \in C^{2}[0,1]$ be a solution of $B V P(12)-(13)$. Since $u^{\prime \prime} \in C[0,1]$, Def. (2.3) show that ${ }^{c} D_{0^{+}}^{\alpha-2} u \in C[0,1]$ and ${ }^{c} D_{0^{+}}^{\alpha-1} u \in C^{1}[0,1]$

From the relation ${ }^{c} D_{0^{+}}^{\alpha} u=g(t)-2 r^{2}{ }^{c} D_{0^{+}}^{\alpha-1} u-r{ }^{c} D_{0^{+}}^{\alpha-2} u$ and $g \in C[0,1]$, we have ${ }^{c} D_{0^{+}}^{\alpha} u \in C(0,1)$. Thus, by Lemma 2.7, we have the following relations

$$
\begin{gathered}
I_{0^{+}}^{\alpha}{ }^{c} D_{0^{+}}^{\alpha} u(t)=u(t)-a_{0}-a_{1} t-a_{2} t^{2}, \quad t \in(0,1) \\
I_{0^{+}}^{\alpha-1}{ }^{c} D_{0^{+}}^{\alpha-1} u(t)=u(t)-b_{1}-b_{2} t, \quad t \in(0,1)
\end{gathered}
$$

and

$$
I_{0^{+}}^{\alpha-2}{ }^{c} D_{0^{+}}^{\alpha-2} u(t)=u(t)-c_{2}, \quad t \in(0,1)
$$

therefore

$$
\begin{gathered}
I_{0^{+}}^{\alpha}{ }^{c} D_{0^{+}}^{\alpha-1} u(t)=I_{0^{+}}^{1} \int_{0^{+}}^{\alpha-1}{ }^{c} D_{0^{+}}^{\alpha-1} u(t) \\
=\int_{0}^{t} u(s) d s-b_{0}-b_{1} t-b_{2} \frac{t^{2}}{2} \\
I_{0^{+}}^{\alpha}{ }^{c} D_{0^{+}}^{\alpha-2} u(t)=I_{0^{+}}^{2} I_{0^{+}}^{\alpha-2}{ }^{c} D_{0^{+}}^{\alpha-2} u(t) \\
=\int_{0}^{t} \int_{0}^{r} u(s) d s d r-c_{0}-c_{1} t-c_{2} \frac{t^{2}}{2}
\end{gathered}
$$

now, from (12), (13) , (18) and (19), we have

$$
(t)+2 r \int_{0}^{t} u(s) d s+r^{2} \int_{0}^{t} \int_{0}^{r} u(s) d s d r=d_{0}+d_{1} t+d_{2} \frac{t^{2}}{2}+I_{0+}^{\alpha} g(t)
$$

where $d_{0}, d_{1}, d_{2} \in \mathbb{R}$.

It is easy to see that $\phi(t)=\int_{0}^{t}(t-s)^{\alpha-3} g(s) d s \in C[0,1]$. Since $u^{\prime \prime} \in C[0,1]$, it follows from (20) that

$$
\begin{gathered}
u^{\prime \prime}(t)+2 r u^{\prime}(t)+r^{2} u(t)=d_{2}+\frac{d^{2}}{d t^{2}} I_{0^{+}}^{\alpha} g(t) \\
=d_{2}+\frac{1}{G(\alpha-2)} \int_{0}^{t}(t-s)^{\alpha-3} g(s) d s
\end{gathered}
$$

assuming $v=u e^{r t}$, the formulas (21) yield

$$
v^{\prime \prime}(t)=d_{2} e^{r t}+\frac{e^{r t}}{G(\alpha-2)} \int_{0}^{t}(t-s)^{\alpha-3} g(s) d s
$$

by integrating both sides of (22) twice, we obtain

$$
v(t)=v(0)+v^{\prime}(0) t+\frac{d_{2}\left(e^{r t}-r t-1\right)}{r^{2}}+\int_{0}^{t} \int_{0}^{n} \int_{0}^{m} \frac{e^{r m}(m-\tau)^{\alpha-3}}{G(\alpha-2)} g(\tau) d \tau d m d n
$$

thus, it follows boundary conditions $u(0)=u(1)$ and $u^{\prime}(0)=u^{\prime}(1)$, that

$$
\begin{gathered}
v(1)=v(0) e^{r} \\
v^{\prime}(1)=v^{\prime}(0) e^{r}
\end{gathered}
$$

now, the formulas (24) and (25) imply that

$$
\begin{aligned}
& v(0)=\frac{d_{2}}{r^{2}}+\frac{1}{\left(e^{r}-1\right)^{2} G(\alpha-2)} \int_{0}^{1} \int_{0}^{m} e^{r m}(m-\tau)^{\alpha-3} g(\tau) d \tau d m \\
& +\frac{1}{\left(e^{r}-1\right) G(\alpha-2)} \int_{0}^{1} \int_{0}^{n} \int_{0}^{m} e^{r m}(m-\tau)^{\alpha-3} g(\tau) d \tau d m d n
\end{aligned}
$$


and

$$
v(0)=\frac{d_{2}}{r}+\frac{1}{\left(e^{r}-1\right) \Gamma(\alpha-2)} \int_{0}^{1} \int_{0}^{m} e^{r m}(m-\tau)^{\alpha-3} g(\tau) d \tau d m
$$

respectively. Substituting (26), and (27) into (23), we have

$$
\begin{aligned}
& v(t)=\frac{d_{2}}{r^{2}} e^{r t}+\frac{1+t\left(e^{r}-1\right)}{\left(e^{r}-1\right)^{2} \Gamma(\alpha-2)} \int_{0}^{1} \int_{0}^{m} e^{r m}(m-\tau)^{\alpha-3} g(\tau) d \tau d m \\
& +\frac{1}{\left(e^{r}-1\right) \Gamma(\alpha-2)} \int_{0}^{1} \int_{0}^{1} \int_{0}^{m} \frac{e^{r m}(m-\tau)^{\alpha-3}}{\left(e^{r}-1\right) \Gamma(\alpha-2)} g(\tau) d \tau d m d n \\
& +\frac{1}{\Gamma(\alpha-2)} \int_{0}^{t} \int_{0}^{n} \int_{0}^{m} e^{r m}(m-\tau)^{\alpha-3} g(\tau) d \tau d m d n
\end{aligned}
$$

by differentiating both sides of (23) and using the condition $u^{\prime}(\xi)+r u(\xi), \xi \in(0,1)$, we have

$$
v^{\prime}(\xi)=\left(u e^{r t}\right)_{t=\xi}^{\prime}=u^{\prime}(\xi) e^{r \xi}+r u(\xi) e^{r \xi}=\left[u^{\prime}(\xi)+r u(\xi)\right] e^{r \xi}=\eta e^{r \xi}
$$

thus,

$$
\begin{aligned}
& \frac{d_{2}}{r} e^{r \xi}+\frac{1}{\left(e^{r}-1\right) G(\alpha-2)} \int_{0}^{1} \int_{0}^{m} e^{r m}(m-\tau)^{\alpha-3} g(\tau) d \tau d m \\
& +\frac{1}{G(\alpha-2)} \int_{0}^{\xi} \int_{0}^{m} e^{r m}(m-\tau)^{\alpha-3} g(\tau) d \tau d m \\
& =\eta e^{r \xi} .
\end{aligned}
$$

Hence, it follows from (30) that

$$
\begin{aligned}
& d_{2}=r \eta-\frac{r e^{-r \xi}}{\left(e^{r}-1\right) \Gamma(\alpha-2)} \int_{0}^{1} \int_{0}^{m} e^{r m}(m-\tau)^{\alpha-3} g(\tau) d \tau d m \\
& -\frac{r e^{-r \xi}}{\Gamma(\alpha-2)} \int_{0}^{\tau} \int_{0}^{m} e^{r m}(m-\tau)^{\alpha-3} g(\tau) d \tau d m .
\end{aligned}
$$

Substituting, (31) into (28), the relation (14) is obtained.

Conversely, since $\int_{0}^{t}(t-s)^{\alpha-3} g(s) d s$ is continuous on $[0,1]$, by differentiating both sides of (14), we obtain

$$
\begin{aligned}
& v^{\prime}(t)=\eta e^{r t}-\frac{e^{r(t-\xi)}}{\left(e^{r}-1\right) \Gamma(\alpha-2)} \int_{0}^{1} \int_{0}^{m} e^{r m}(m-\tau)^{\alpha-3} g(\tau) d \tau d m \\
& -\frac{e^{r(t-\xi)}}{\Gamma(\alpha-2)} \int_{0}^{\tau} \int_{0}^{m} e^{r m}(m-\tau)^{\alpha-3} g(\tau) d \tau d m \\
& +\frac{1}{\left(e^{r}-1\right) \Gamma(\alpha-2)} \int_{0}^{1} \int_{0}^{m} e^{r m}(m-\tau)^{\alpha-3} g(\tau) d \tau d m \\
& +\frac{1}{\Gamma(\alpha-2)} \int_{0}^{t} \int_{0}^{m} e^{r m}(m-\tau)^{\alpha-3} g(\tau) d \tau d m .
\end{aligned}
$$

By differentiating both sides of (32), we will get

$$
\begin{aligned}
& v^{\prime \prime}(t)=r \eta e^{r t}-\frac{r e^{r(t-\xi)}}{\left(e^{r}-1\right) \Gamma(\alpha-2)} \int_{0}^{1} \int_{0}^{m} e^{r m}(m-\tau)^{\alpha-3} g(\tau) d \tau d m \\
& -\frac{r e^{r(t-\xi)}}{\Gamma(\alpha-2)} \int_{0}^{1} \int_{0}^{m} e^{r m}(m-\tau)^{\alpha-3} g(\tau) d \tau d m \\
& +\frac{e^{r t}}{\Gamma(\alpha-2)} \int_{0}^{t}(t-\tau)^{\alpha-3} g(\tau) d \tau \\
& =e^{r t}\left(d_{2}+I^{\alpha-2} g(t)\right)
\end{aligned}
$$

where $d_{2}$ is described as in (31), and so $v \in C^{2}[0,1]$. Furthermore, from (32) together with (23) and $(31)$, we ensure that $(24)$ holds on $[0,1]$, and

$$
v(1)=v(0) e^{r}, v^{\prime}(1)=v^{\prime}(0) e^{r}, v^{\prime}(\xi)=\eta e^{r \xi}, \quad \xi \in(0,1),
$$


Now, assume that $u=v e^{-r t}$. Keeping in mind that $u^{\prime \prime} \in C[0,1]$, because $v \in C^{2}[0,1]$, it follows (32) and (33) that

$$
u^{\prime \prime}+2 r u^{\prime}+r^{2} u=d_{2}+I_{0^{+}}^{\alpha-2} g(t) .
$$

Therefore,

$$
{ }^{c} D_{0^{+}}^{\alpha-2} u^{\prime \prime}+2 r^{c} D_{0^{+}}^{\alpha-2} u^{\prime}+r^{2}{ }^{c} D_{0^{+}}^{\alpha-2} u={ }^{c} D_{0^{+}}^{\alpha-2} I_{0^{+}}^{\alpha-2} g(t)=g(t),
$$

on $(0,1)$. From the fact that $u^{\prime \prime} \in C[0,1]$, and Def. (2.4) we get

$$
\begin{aligned}
& { }^{c} D_{0^{+}}^{\alpha} u(t)=\left[D_{0^{+}}^{\alpha}\left\{u(s)-u(0)-u^{\prime}(0) s-\frac{u(0)}{2} s^{2}\right\}\right](t) \\
& =\left[D_{0^{+}}^{\alpha} u\right](t)-\frac{u(0)}{G(1-\alpha)} t^{-\alpha}-\frac{u^{\prime}(0)}{G(2-\alpha)} t^{1-\alpha}-\frac{u^{\prime \prime}(0)}{G(3-\alpha)} t^{2-\alpha},
\end{aligned}
$$

and

$$
\begin{aligned}
{\left[D_{0^{+}}^{\alpha} u\right](t)=} & \frac{1}{G(3-\alpha)} \frac{d^{3}}{d t^{3}} \int_{0}^{t}(t-s)^{2-\alpha} u(s) d s \\
= & \frac{1}{(\alpha-3) G(3-\alpha)} \frac{d^{3}}{d t^{3}} \int_{0}^{t} u(s) d(t-s)^{3-\alpha} \\
= & \frac{1}{(3-\alpha) G(3-\alpha)} \frac{d^{3}}{d t^{3}}\left\{u(0) t^{3-\alpha}+\int_{0}^{t}(t-s)^{3-\alpha} u^{\prime}(s) d s\right\} \\
= & \frac{1}{(\alpha-4)(3-\alpha) G(3-\alpha)} \frac{d^{3}}{d t^{3}}\left\{(\alpha-4) u(0) t^{3-\alpha}\right. \\
= & \left.\frac{1}{G(5-\alpha)} \frac{d^{3}}{d t^{3}} \int_{0}^{t} u^{\prime}(s) d(t-s)^{4-\alpha}\right\} \\
= & \frac{1}{G(5-\alpha)}\left\{\int_{0}^{t}(t-s)^{4-\alpha} u_{j=0}^{1}\left[\prod_{i=j+1}^{4}(i-\alpha) d s\right\}\right. \\
& \left.+\frac{d}{d t} \int_{0}^{t}(t-s)^{2-\alpha} u^{\prime \prime}(s) d s\right\} .
\end{aligned}
$$

Consequently,

$$
\left[D_{0^{+}}^{\alpha} u\right](t)=\frac{u(0)}{\Gamma(1-\alpha)} t^{-\alpha}-\frac{u^{\prime}(0)}{\Gamma(2-\alpha)} t^{1-\alpha}+\frac{1}{\Gamma(3-\alpha)} \frac{d}{d t} \int_{0}^{t}(t-s)^{2-\alpha} u^{\prime \prime}(s) d s .
$$

It follows (37) and (38) that

$$
\begin{aligned}
{\left[{ }^{c} D_{0^{+}}^{\alpha} u(s)\right](t) } & =\left[D_{0^{+}}^{\alpha-2} u^{\prime \prime}(s)\right](t)-\frac{u^{\prime \prime}(0)}{G(3-\alpha)} t^{2-\alpha} \\
& =D_{0^{+}}^{\alpha-2}\left[u^{\prime \prime}(s)-u^{\prime \prime}(0)\right](t) \\
& =\left[{ }^{c} D_{0^{+}}^{\alpha} u^{\prime \prime}(s)\right](t) .
\end{aligned}
$$

Similarly, we can show that $\left[{ }^{c} D_{0^{+}}^{\alpha-1} u\right](t)=\left[{ }^{c} D_{0^{+}}^{\alpha-2} u^{\prime}\right](t)$. Moreover, it follows (36) that

$$
{ }^{c} D_{0^{+}}^{\alpha} u(t)+2 r^{c} D_{0^{+}}^{\alpha-1} u(t)+r^{2}{ }^{c} D_{0^{+}}^{\alpha-2} u(t)=g(t), \quad t \in(0,1) .
$$

On the other hand, the relation (34) implies that

$$
u(0)=u(1), \quad u^{\prime}(0)=u^{\prime}(1), \quad u^{\prime}(\xi)+r u(\xi)=\eta .
$$

Then, $u \in C^{2}[0,1]$ is a solution of $B V P(12)-(13)$. Thus, this ends the proof. 


\section{Main Result}

Let $U=C[0,1]$ be a Banach space with the norm $\|u\|=\max _{t \in[0,1]}\{u(t)\}$. Consider the space $U$ with the norm $\|u\|_{*}=\max _{t \in[0,1]}\left\{u(t) e^{-r t}\right\}$ in which $r$ is described as in (1). It is well known that the norm $\|u\|_{*}$ is equivalent to the norm $\|u\|$.

For the forthcoming analysis, we need the assumptions $(A 0)$ and $(A 1)$.

Theorem 3.1. Let the assumption $(A 0)$ hold. Then, the boundary value problem $(1)-(2)$ has a unique solution.

Proof. Define the operator $T: U \rightarrow U$ by

$$
\begin{aligned}
& \operatorname{Tv}(t)=\frac{\eta}{r} e^{r t}-\frac{e^{r(t-\xi)}}{r\left(e^{r}-1\right) \Gamma(\alpha-2)} \int_{0}^{1} \int_{0}^{m} e^{r m}(m-\tau)^{\alpha-3} g(\tau) d \tau d m \\
& -\frac{e^{r(t-\xi)}}{r \Gamma(\alpha-2)} \int_{0}^{\xi} \int_{0}^{m} e^{r m}(m-\tau)^{\alpha-3} g(\tau) d \tau d m \\
& +\frac{1+t\left(e^{r}-1\right)}{\left(e^{r}-1\right)^{2} \Gamma(\alpha-2)} \int_{0}^{1} \int_{0}^{m} e^{r m}(m-\tau)^{\alpha-3} g(\tau) d \tau d m \\
& +\int_{0}^{1} \int_{0}^{1} \int_{0}^{m} \frac{e^{r m}(m-\tau)^{\alpha-3}}{\left(e^{r}-1\right) \Gamma(\alpha-2)} g(\tau) d \tau d m d n \\
& +\int_{0}^{t} \int_{0}^{n} \int_{0}^{m} \frac{(m-\tau)^{\alpha-3}}{\Gamma(\alpha-2)} g(\tau) d \tau d m d n
\end{aligned}
$$

where the function $g(t)=f\left(t, v(t) e^{-r t}\right)$ is continuous on $[0,1]$, for any $v \in U$ (from $\left.\left(A_{0}\right)\right)$. It is easy to see that the operator $T$ maps $U$ into $U$.

In view of Lemma (2.10), the operator $T$ has a fixed point $v \in V$ if and only if $u=v e^{-r t}$ is a solution of $B V P(1.1)-(1.2)$ with $u \in C^{2}[0,1]$. So, it is sufficient to show that the operator $T$ has a fixed point on $U$. For $v_{1}, v_{2} \in U$ and for $s \in C[0,1]$, we obtain

$$
\begin{gathered}
\left|f\left(s, v_{2}(s) e^{-r s}\right)-f\left(s, v_{1}(s) e^{-r s}\right)\right| \leqslant L\left|v_{2}(s) e^{-r s}-v_{1}(s) e^{-r s}\right| \\
\leqslant L\left\|v_{2}-v_{1}\right\|_{*}
\end{gathered}
$$

Hence, from (39), we have the following inequality

$$
\begin{aligned}
& \left|T v_{2}(t)-T v_{1}(t)\right| \leqslant \frac{L}{G(\alpha-2)}|| v_{2}-v_{1} \|_{*} \\
& {\left[\frac{e^{r(t-\xi)}}{r\left(e^{r}-1\right)} \int_{0}^{1} \int_{0}^{m} e^{r m}(m-\tau)^{\alpha-3} d \tau d m\right.} \\
& +\frac{e^{r(t-\xi)}}{r} \int_{0}^{\xi} \int_{0}^{m} e^{r m}(m-\tau)^{\alpha-3} d \tau d m \\
& +\frac{1+t\left(e^{r}-1\right)}{\left(e^{r}-1\right)^{2}} \int_{0}^{1} \int_{0}^{m} e^{r m}(m-\tau)^{\alpha-3} d \tau d m \\
& +\frac{1}{e^{r}-1} \int_{0}^{1} \int_{0}^{n} \int_{0}^{m} e^{r m}(m-\tau)^{\alpha-3} d \tau d m d n \\
& \left.+\int_{0}^{t} \int_{0}^{n} \int_{0}^{m} e^{r m}(m-\tau)^{\alpha-3} d \tau d m d n\right] \\
& \leqslant \frac{2 L e^{r t}}{r^{2} \Gamma(\alpha-1)}\left\|v_{2}-v_{1}\right\|_{*} .
\end{aligned}
$$

Consequently,

$$
\left\|T v_{2}(t)-T v_{1}(t)\right\|_{*} \leqslant \frac{2 L e^{r t}}{r^{2} \Gamma(\alpha-1)}\left\|v_{2}-v_{1}\right\|_{*} .
$$

By the Banach contraction principle, it follows that $T$ has an unique fixed point $v \in U$. Therefore, $u=e^{-r t} v$ is a unique solution of $F B V P(1)-(2)$.

Now, we prove the existence of solutions of $(1)-(2)$ by applying Schauder fixed point theorem. 
Theorem 3.2. Let the assumption $(A 1)$ hold. Then, the boundary value problem (1) - (2) has at least one solution $u \in C^{2}[0,1]$.

Proof. Let us consider $P=\sup \{|p(t)| ; t \in[0,1]\}$ and $B_{R}=\left\{v \in U ;\left\|v-v_{0}\right\|_{*} \leqslant R\right\}$ in which $v_{0}=\frac{\eta}{r} e^{r t}$ and $R>\frac{2(P r+A \eta)}{r\left(r^{2} \Gamma(\alpha-1) 2 A\right)}$. For $v \in U$, by $(A 1)$, we find that

$$
\left|f\left(s, v(s) e^{-r s}\right)\right| \leqslant P+A\|v\|_{*} \leqslant P+A\left(\left\|v_{0}\right\|_{*}+R\right) \leqslant P+A\left(\frac{\eta}{r}+R\right),
$$

and so,

$$
\left|T v(t)-v_{0}(t)\right| \leqslant \frac{P+A\left(\frac{\eta}{r}+R\right)}{r^{2} \Gamma(\alpha-1)}\left[e^{r(t-\xi)}+e^{r(t-\xi)}\left(e^{r \xi}-1\right)+e^{r t}\right] .
$$

From (41), we have

$$
\left\|T v-v_{0}\right\|_{*} \leqslant \frac{2\left[P+A\left(\frac{\eta}{r}+R\right)\right]}{r^{2} G(\alpha-1)}<R .
$$

Thus, $T$ maps $B_{R}$ into $B_{R}$, i.e. $T\left(B_{R}\right) \subseteq B_{R}$. Now, we prove that $T$ is completely continuous on $B_{R}$. We will give the proof in the case that $U$ is equipped with the usual norm, since the norm $\|u\|_{*}$ is equivalent to the usual norm. Since $T\left(B_{R}\right) \subseteq B_{R}$, we have $\|T u\| \leqslant\|T u\|_{*} e^{r} \leqslant\left(v_{0}+R\right) e^{r}$ for any $u \in B_{R}$, and so $\left\{z ; z \in T\left(B_{R}\right)\right\}$ is uniformly bounded. On the other hand, for any $v \in B_{R}$, it follows from (40) and $(A 1)$ that

$$
(T v)^{\prime}(t) \leqslant\left(\eta+\frac{P+A\left(\frac{\eta}{r}+R\right)}{r G(\alpha-1)}\right) e^{r}, \quad t \in[0,1]
$$

and this shows that $T\left(B_{R}\right)$ is equicontinuous. Thus, by Arzella-Ascoli theorem, it implies that $T\left(B_{R}\right)$ is relatively compact. Finally, we show that $T$ is continuous on $B_{R}$. Let $\left(v_{n}\right)$ be an arbitrary sequence in $B_{R}$ and $v \in B_{R}$ so that $\left\|v_{n}-v\right\| \rightarrow 0$ as $n \rightarrow \infty$. Therefore, $\left\|v_{n}-v\right\|_{*} \rightarrow 0$, as $n \rightarrow \infty$ and so there exists two constants $k_{1}, k_{2}$ so that $v_{n}(t) e^{-r t}(n=1,2, \ldots)$ and $v(t) e^{-r t} \in\left[k_{1}, k_{2}\right]$, for each $t \in[0,1]$. Since $f$ is uniformly continuous on $[0,1] \times\left[k_{1}, k_{2}\right]$, it follows that for any $\epsilon>0$, there exists $\delta>0$ whenever $\left|u_{1}-u_{2}\right|<\delta, u_{1}, u_{2} \in\left[k_{1}, k_{2}\right]$ then,

$$
\left|f\left(t, u_{2}\right)-f\left(t, u_{1}\right)\right| \leqslant \vartheta \epsilon, \quad t \in[0,1]
$$

where $\vartheta=\frac{r^{2} \Gamma(\alpha-1)}{2 e^{r}}$. Since $v_{n} \rightarrow v$, there exists $N \geqslant 1$, such that the following relation

$$
\left|v_{n}(t) e^{-r t}-v(t) e^{-r t}\right| \leqslant \delta, \quad t \in[0,1]
$$

satisfies for $n \geqslant N$. Now, for any $n \geqslant N(3.5)$ yields

$$
\begin{aligned}
& \left|T v_{n}(t)-T v(t)\right| \leqslant \frac{\vartheta \epsilon}{G(\alpha-2)}\left[\frac{e^{r(t-\xi)}}{r(\alpha-1)} \int_{0}^{1} \int_{0}^{m} e^{r m}(m-s)^{\alpha-3} d \tau d m\right. \\
& +\frac{e^{r(t-\xi)}}{r} \int_{0}^{\xi} \int_{0}^{m} e^{r m}(m-s)^{\alpha-3} d \tau d m \\
& +\frac{1+t\left(e^{r}-1\right)}{\left(e^{r}-1\right)^{2}} \int_{0}^{1} \int_{0}^{m} e^{r m}(m-\tau)^{\alpha-3} d \tau d m \\
& +\frac{1}{e^{r}-1} \int_{0}^{1} \int_{0}^{n} \int_{0}^{m} e^{r m}(m-\tau)^{\alpha-3} d \tau d m d n \\
& \left.+\int_{0}^{t} \int_{0}^{n} \int_{0}^{m} e^{r m}(m-\tau)^{\alpha-3} d \tau d m d n\right] \\
& \leqslant \frac{2 \vartheta \epsilon}{r^{2} \Gamma(\alpha-1)} e^{r t} .
\end{aligned}
$$


Consequently

$$
\left\|T v_{\mathrm{n}}(t)-T v(t)\right\|<\frac{2 \vartheta \epsilon}{r^{2} \Gamma(\alpha-1)} e^{r}=\epsilon .
$$

Thus, all the assumptions of the Schauder fixed point theorem are satisfied. Then, there exists a point $v \in B_{R}$ with $v=T v$ In view of Lemma (14), we conclude that $u=v e^{-r t}\left(u \in C^{2}[0,1]\right)$ is a solution of boundary value problem $(1)-(2)$. As a result, the proof is complete.

\section{Illustrative Examples}

Example 4.1. Consider the boundary value problem

$$
\left\{\begin{array}{c}
{ }^{c} D_{0^{+}}^{\frac{5}{2}} u(t)+2 r^{c} D_{0^{+}}^{\frac{3}{2}} u(t)+r^{2} u(t)=f(t, u(t)), \quad 0<t<1, \\
u(0)=u(1), \quad u^{\prime}(0)=u^{\prime}(1), \quad u^{\prime}(\xi)+r u(\xi)=\eta
\end{array}\right.
$$

where $r>0, f(t, u)=h(t) \frac{u}{1+u^{2}}$ with

$$
|h(t)| \leqslant \frac{r^{2} G\left(\frac{5}{2}-1\right)}{2 e^{r}}=\frac{r^{2} \sqrt{\pi}}{4 e^{r}} .
$$

It is easy to see that the assumption $(A \boldsymbol{o})$ holds. So, by Theorem 3.1, $B V P(43)$ has a unique solution.

Example 4.2. Consider the boundary value problem

$$
\left\{\begin{array}{c}
{ }^{c} D_{0^{+}}^{\frac{5}{2}} u(t)+2 r^{c} D_{0^{+}}^{\frac{3}{2}} u(t)+r^{2} u(t)=f(t, u(t)), \quad 0<t<1, \\
u(0)=u(1), \quad u^{\prime}(0)=u^{\prime}(1), \quad u^{\prime}(\xi)+r u(\xi)=\eta
\end{array}\right.
$$

where $r>0, f(t, u)=p_{1}(t)+p_{2}(t) u$ with $p_{1}, p_{2} \in C[0,1]$ and $\max \left|p_{2}(t)\right|_{t \in[0,1]} \leqslant \frac{r^{2} \sqrt{\pi}}{4}$. Thus, the conclusion of Theorem 3.2 applies to the problem.

Acknowledgments: The authors are grateful to the referees for their comments and suggestions which improved the quality of the paper.

Author Contributions: All authors contributed to the technical analysis and development of the results.

Conflicts of Interest: The authors declare no conflict of interest.

\section{References}

1. Oldham, K.B.; Spanier, J. The Fractional Calculus; Academic press: New York, NY, USA; London, UK, 1974.

2. The Fractional Calculus and Its Application; Ross, B., Ed.; Lecture notes in mathematics; Springer-Verlag: Berlin, Germany, 1975.

3. Tatom, F.B. The relationship between fractional calculus and fractals. Fractals 1995, 3, 217-229. [CrossRef]

4. Nonnenmacher, T.F.; Metzler, R. On the Riemann-Liouville fractional calculus and some recent applications. Fractals 1995, 3, 557-566. [CrossRef]

5. Samko, S.G.; Kilbas, A.A.; Marichev, O.I. Fractional Integral and Derivatives: Theory and Application; CRC Press: Boca Raton, FL, USA, 1993.

6. Kilbas, A.A.; Srivastava, H.M.; Trujillo, J.J. Theory and Application of Fractional Differential Equations; Elsevier Science: Amsterdam, The Netherlands, 2006.

7. Miller, K.S.; Ross, B. An Introduction to the Fractional Calculus and Fractional Differential Equation; John Wiley and Sons: New York, NY, USA, 1993.

8. Podlubny, I. Fractional Differential Equations; Academic Press: San Diego, CA, USA, 1999.

9. Lakshmikantham, V.; Leela, S.; Vasundhara, J. Theory of Fractional Dynamic Systems; Cambridge Academic Publishers: Cambridge, UK, 2009. 
10. Agarwal, R.P.; Benchohara, M.; Slimani, B.A. Existence results for differential equations with fractional order and impulses. Mem. Diff. Equ. Math. Phys. 2008, 44, 1-21.

11. Agarwal, R.P.; Benchohara, M.; Hamani, S. Boundary value problems for fractional differential equations. J. Geor. Math. 2009, 16, 401-411.

12. Ahmad, B.; Nieto, J.J. Existence of solutions for nonlocal boundary value problems of higher order nonlinear fractional differential equations. Abstr. Appl. Anal. 2009. [CrossRef]

13. Darzi, R.; Mohammadzadeh, B.; Neamaty, A.; Băleanu, D. Lower and upper solutions method for positive solutions of fractional boundary value problems. Abstr. Appl. Anal. 2013. [CrossRef]

14. Zhang, S. Positive solutions for boundary value problems of nonlinear fractional differential equations. Elect. J. Diff. Equ. 2006, 36, 1-12. [CrossRef]

15. Rehman, M.U.; Khan, R.A. Existence and uniqueness of solutions form multi-point for boundary value problems for fractional differential equations. Appl. Math. Lett. 2010, 23, 1038-1044. [CrossRef]

16. Darzi, R.; Mohammadzadeh, B.; Neamaty, A.; Băleanu, D. On the Existence and Uniqueness of Solution of a Nonlinear Fractional Differential Equations. J. Comput. Anal. Appl. 2013, 15, 152-162.

17. Băleanu, D.; Diethelm, K.; Scalas, E.; Trujillo, J.J. Fractional Calculus: Models and Numerical Methods; World Scientific: Singapore, Singapore, 2012.

18. Băleanu, D.; Trujillo, J.J. On exact solutions of a class of fractional Euler-Lagrange equations. Nonlinear Dyn. 2008, 52, 331-335. [CrossRef]

19. Băleanu, D.; Mustafa, O.G. On the global existence of solutions to a class fractional differential equations. Comp. Math. Appl. 2010, 59, 1835-1841. [CrossRef]

20. El-Shahed, M. Nontrivial solutions for a nonlinear multi-point boundary value problems of fractional order. Comp. Math. Appl. 2010, 59, 3438-3443. [CrossRef]

21. Chai, G. Existence results for boundary value problems of nonlinear fractional differential equations. Comp. Math. Appl. 2011, 62, 2374-2382. [CrossRef]

22. Anguraj, A.; Ranjini, M.C.; Rivero, M.; Trujillo, J.J. Existence results for fractional neutral functional differential equations with random impulses. Mathematics 2015, 3, 16-28. [CrossRef]

23. Sambandham, B.; Vatsala, A.S. Basic results for sequential Caputo fractional differential equations. Mathematics 2015, 3, 76-91. [CrossRef]

24. Morita, T.; Sato, K. Asymptotic expansions of fractional derivatives and their applications. Mathematics 2015, 3, 171-189. [CrossRef]

25. Diekema, E. The fractional orthogonal derivative. Mathematics 2015, 3, 273-298. [CrossRef]

26. Rogosin, S. The role of the Mittag-Leffler function in fractional modeling. Mathematics 2015, 3, 368-381. [CrossRef]

27. Nieto, J.J.; Ouahab, A.; Venktesh, V. Implicit fractional differential equations via the Liouville-Caputo derivative. Mathematics 2015, 3, 398-411. [CrossRef]

(C) 2016 by the authors; licensee MDPI, Basel, Switzerland. This article is an open access article distributed under the terms and conditions of the Creative Commons by Attribution (CC-BY) license (http:/ / creativecommons.org/licenses/by/4.0/). 